\title{
Evaluation of the Use of Artificial Neural Networks to Predict the Photovoltaic Power Generation Factors by Using Feed Forward Back Propagation (FFBP) Technique
}

\author{
Shreeniket Trivedi \\ Electrical and Electronics Engineering, National Institute of Technology, Puducherry, India
}

\begin{abstract}
The solar power generation (renewable energy) is the cleanest form of energy generation method and the solar power plant has a very long life and also is maintenance free, but due to the high unpredictability of the generated solar power due to dynamically changing environmental factors it cannot be used as the reliable source of power. This prevents the maximum utilization of solar energy. In this project we are designing the artificial neural network model to predict the power generated depending on the various environmental factors like visibility, cloud cover (sky cover), etc. the intensity of the incident of the solar radiation decreases and thus the plant is not able to work at its rated capacity. We use Artificial Neural Network (ANN) with Feed Forward Back Propagation (FFBP) technique and predicted the percentage of the maximum plant capacity which will be generated by considering the environmental factors like temperature, pressure, distance to solar noon, day light, sky cover, visibility, humidity, wind speed, wind direction and compared our results with available data and find quite encouraging results.
\end{abstract}

KEYWORDS: Solar power, photovoltaic, ANN, FFBP, environmental factors.

\section{INTRODUCTION}

The photovoltaic energy generation is the cheapest and cleanest form of energy generation method. Recently, solar Photovoltaic (PV) generation systems are getting more worldwide adoption due to wider availability of solar energy, improvement in panel efficiency, extended life time [1]. The importance of solar energy is marked by the fact that solar generation is one of the most common types of renewable energy that has grown rapidly over the past decade, and it is expected to grow even faster in the future [2]. To match the load and the generation of the central power grid we must connect the power generation plant to the central grid in the most economically efficient and reliable manner. Thus an accurate prediction of power generation in the solar energy plant is essentially required. Consequently, it is important to determine parameters that can help in best possible prediction in regard of photovoltaic power generated [3]. A plentiful models have been proposed for this purpose with high accuracy rates. However, identifying weather factors which influence the solar power prediction most are less explored.

In this paper, two broad section is studied that can help best predict solar power, firstly, the study of the environmental factor that affect the production of solar power by solar cell and the study of the working of Artificial Neural Network (ANN) to predict photovoltaic energy generation. Our project works on the goal to make solar power plant a reliable source of power.

\section{TECHNIQUEUSED TO DESIGN THE MODEL}

In our working model design we have taken environmental data as inputs and used Feed Forward Back Propagation (FFBP) technique for training the ANN model to predict the probability of generated power out of total capacity of solar power plant.

\section{Environmental factors as input data:}

The unpredictable behaviour of the climate affects the power output and causes an unfavourable impact on the stability, reliability and operation of the grid. Performance of the Solar Photovoltaic System has substantial influence of environmental factors [4] also velocity of air and presence of dust, humidity significantly affect the efficiency of photovoltaic cell [5].

Ramadan J. Mustafa et al. in 2020 for the first time used simultaneously four environmental factors (the accumulation of dust, water droplets, birds' droppings, and partial shading conditions) affecting system performance. The results obtained from this investigation demonstrate that the accumulation of dust, shading, and bird fouling has a significant effect on PV current and voltage [6].

In present study a relation between solar power generation and water droplet, partial shading, dust accumulation, solar radiation, 


\section{International Journal of Current Science Research and Review}

ISSN: 2581-8341

Volume 04 Issue 02 February 2021

DOI: 10.47191/ijcsrr/V4-i2-06, Impact Factor: 5.825

IJCSRR @ 2021

Www.ijcsrr.org

bird droppings, temperature and humidity are formulated. In our formulation the partial shading and the dust are kept under the visibility parameter. Whereas water droplet and birds dropping are kept under the sky cover parameter. Since, solar radiation has a direct effect on the temperature. Thus, it is replaced with temperature parameter. In present study temperature, pressure, distance to solar noon, day light, sky cover, visibility, humidity, wind speed and wind direction are measured as input environmental parameters to predict the generated solar power.

\section{Technique of ANN Model:}

Because of their ability to generate non-linear mappings during training, ANNs are particularly well suited to complex, real-world problems such as understanding climate [7]. Neural networks are highly non-convex models with extreme capacity that train fast and generalize well [8]. The major milestones of neural networks date back to McCulloch and Pitts [9], Widrow and Hoff [10].

In last decade many researchers has used ANN for solar data prediction in various parts of world. In 2011 a Nigerian researcher Abdul Azeez evaluated ANN for global solar radiation [11], in the same year Chen et al. also used this method to forecast the power of PV panels and find it accurate and efficient in the operation of a PV system [12]. In 2010 Paoli et al. [13] and in 2011 LinaresRodr' 'guez et al. [14] used a Multilayer Perceptron (MLP) for solar radiation prediction and explained a multilayer perceptron feed forward neural network giving emphasis to that the high ability of an ANN for prediction. Though ANN and its different related models are shown to be popular among researches but only a few describe forecasting models used to predict directly the daily energy production of the PV plant [15].

Among the different forms of ANN, Feed Forward Back Propagation and logistic regression neural networks are proposed in this research. FFBP technique is used for training the ANN model with ten meteorological factors (temperature, pressure, distance to solar noon, day light, sky cover, visibility, humidity, wind speed, wind speed period and wind direction) as input parameters in the model for predicting the probability of generated power out of total capacity of solar power plant. The weights of input combinations and bias values were optimized using back propagation algorithm. The model performances were tested using available benchmark data of actual generated power at specific metrological condition for evaluating the ANN models. The observed data of the simulation and experimental test were analyzed using MATLAB.

In Feed Forward (FF) neural network the information flow is in the forward direction. The Feed Forward Neural Network (FFNN) is defined as a type of multilayer perceptron (MLP) network with special input values. These values that are multiplied by their weight are led to the hidden layer by neurons. Corani in 2005 utilized a FFNN for air prediction in Milan [16]. He described the structure of the FFNN in different three layers. An input layer was used for data collection, a hidden layer for data processing by neurons and the output layer for the results.

In the mid-1980s. Werbos [17], Parker [18] and Rumelhart. [19] Proposed the back-propagation algorithm. Back propagation (BP) is assumed as a popular learning technique for Feed Forward neural networks [20]. In prediction models the Back Propagation Algorithm (BPA) or the generalized delta rule is also termed as Supervised Learning Algorithm (SLA) that aims at reducing overall system error to a minimum [21]. Therefore, the FFBP is a well-defined model for forecasting parameters. A schematic diagram of prediction model using FFBP is shown in Figure-1.

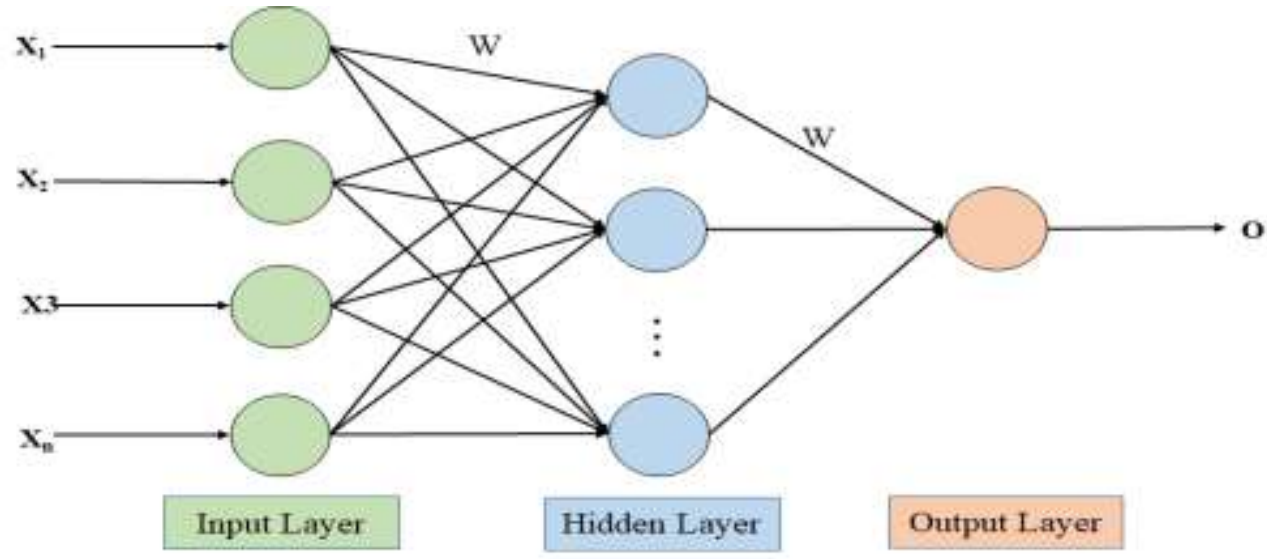

Figure-1: Prediction Using FFBP 


\title{
International Journal of Current Science Research and Review
}

ISSN: 2581-8341

\author{
Volume 04 Issue 02 February 2021
}

DOI: 10.47191/ijcsrr/V4-i2-06, Impact Factor: 5.825

IJCSRR@ 2021

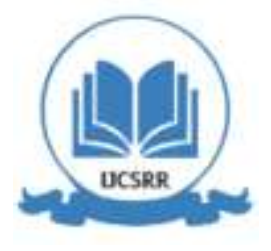

www.ijcsrr.org

In 2010 Mellit and Pavan [23] provided a feed forward neural network for solar radiance forecasting in Italy. In 2012 Kumari et al. used a seasonal autoregressive integrated moving average (SARIMA) model, feed forward neural networks (FFNN) for air temperature prediction in India [24]. Khatib et al. in 2012 developed a feed forward multilayer perception model of ANN with four inputs for solar prediction in Malaysia [25] with reduced the mean absolute percentage error (MAPE) of 9.8\%. The ANN in that study was a feed forward back propagation model. The results proved that this method is precise and suitable for forecasting solar radiation. The back-propagation algorithm helps to train the ANN to recognize similar patterns [26] [27].

In this model the training process for the back propagation algorithm, first, the random weights are initialized for the neurons and the output is measured. The error is calculated by comparing measured output with the desired value. Based on the calculated error using the partial differentiation of the loss function, the bias and weights are changed in the second step. Thus, to update the weights of the previous layers the error is propagated backward [22]. These steps are repeated for 10, 100, 1000, 10000 and 100000 iterations and the error vs error frequency is plotted for each of them.

\section{COMPUTATION USING MATLAB CODE}

\section{Data Preparation:}

In the data preparation process raw data are search form an open-source website called "Kaggle" and downloaded. This raw data is cleaned to remove the missing data and suitably processed as per our requirements. Next step is visualization of the data. In this step processed data is used for the graph formation between various input and output parameters. After this step the data is split into two parts. First part is called the training data and is eighty five percent of the total dataset. This training data is used to train the ANN model. Remaining fifteen percent data is called the testing data and it will be used to test the trained ANN model. Flowchart of Data Processing are depicted in Figure-2 Below

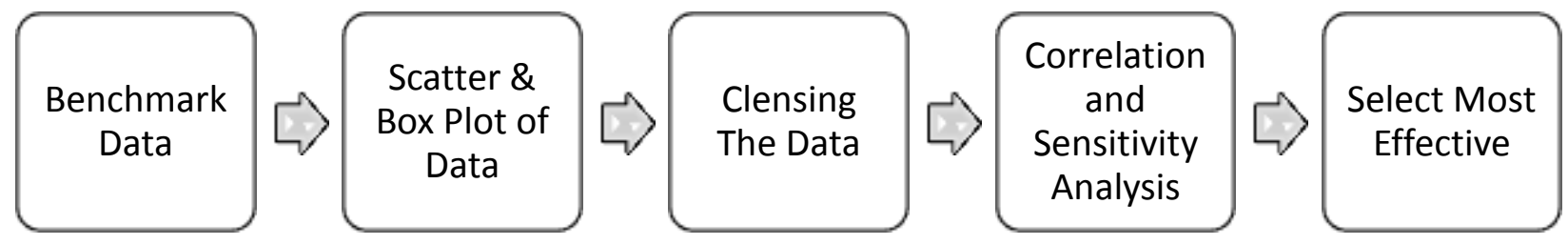

Fig-2. Flowchart of Data Processing

\section{Model Training:}

In this project we use the logistic regression model of ANN. The activation function used is Binary sigmoid activation function which limits the output range between 0 and 1 and gives the percentage of the total capacity of the power plant that is predicted to be generated at that particular input. The back propagation is done as grading descend function taking the value of learning rate as 0.0001.The training model is run for multiple number of iterations maximum of which is one lakh. For the different number of iterations, the trained model is tested and error v/s error frequency is plotted.

\section{Model Testing:}

In this step the trained model is tested using testing dataset. The input parameters of the dataset are given as an input to the trained model following which the trained model predicts the output. The predicted output is then compared with the actual output. The difference between the predicted and the actual value is calculated. This is the error in the prediction. Then the frequency of the error $\mathrm{v} / \mathrm{s}$ error value is plotted.

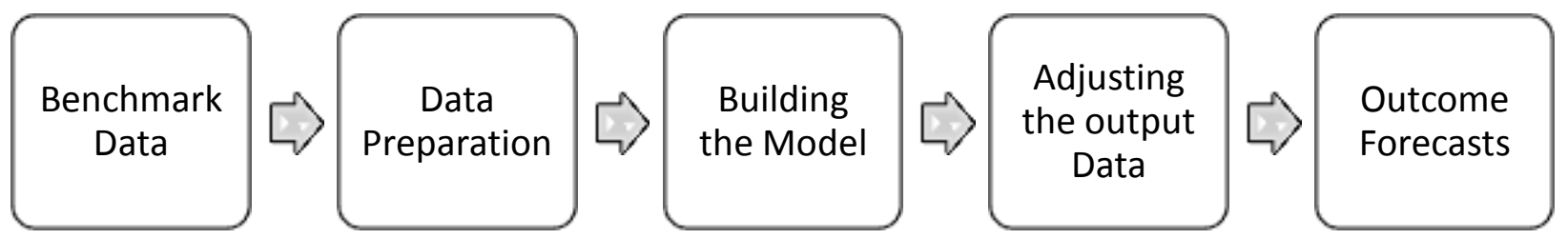

Fig-3. Flowchart of Solar Forecasting Model 
International Journal of Current Science Research and Review

ISSN: 2581-8341

Volume 04 Issue 02 February 2021

DOI: 10.47191/ijcsrr/V4-i2-06, Impact Factor: 5.825

IJCSRR@ 2021

www.ijcsrr.org

Waveforms:

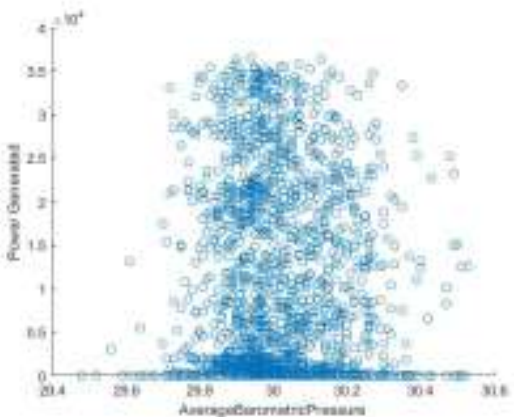

Fig-4. Power Generated v/s Pressure

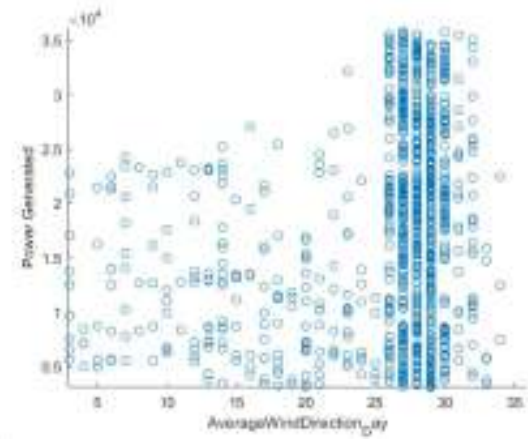

Fig-6. Power Generated v/s Wind Direction

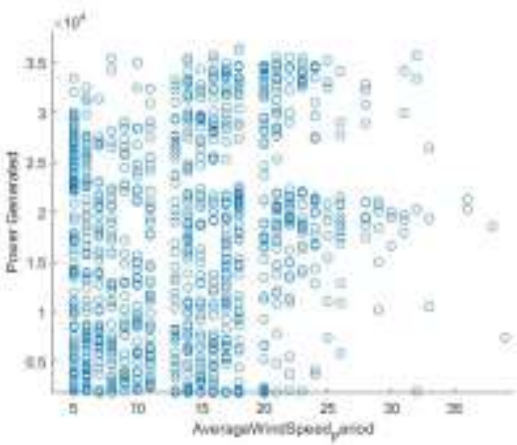

Fig-8. Power Generated v/s Wind Speed Period

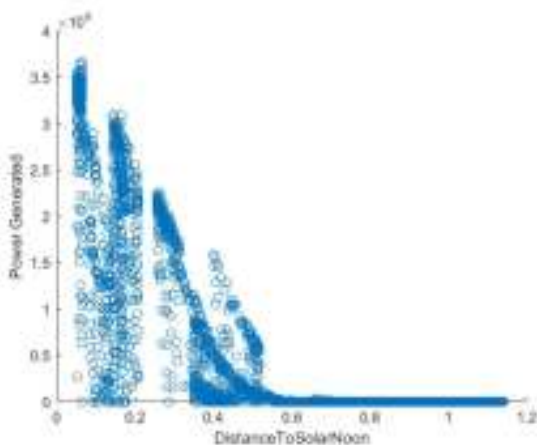

Fig-10. Power Generated v/s Distance to Solar Noon

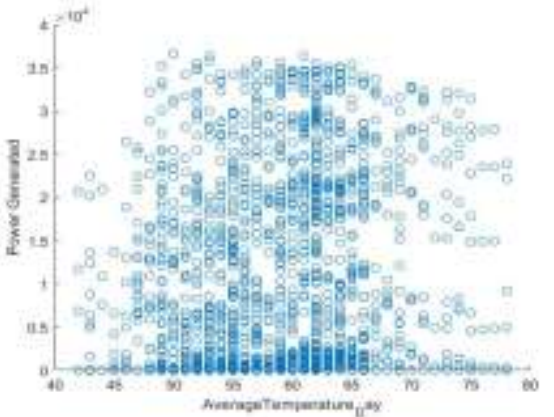

Fig-5. Power Generated v/s Temperature

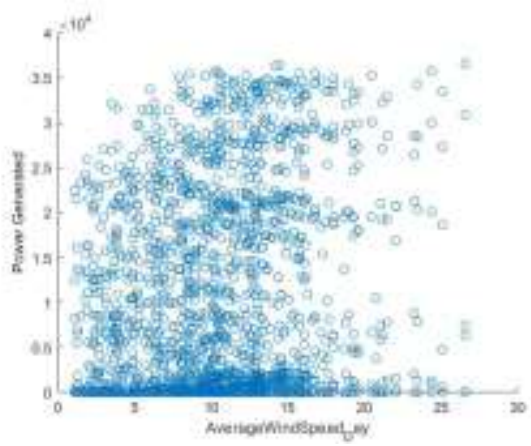

Fig-7. Power Generated v/s Wind Speed

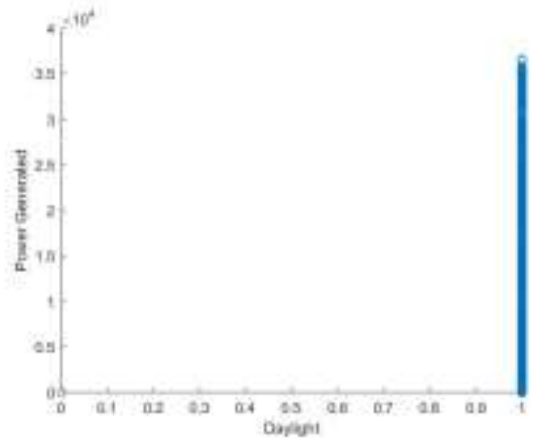

Fig-9. Power Generated v/s Day Light

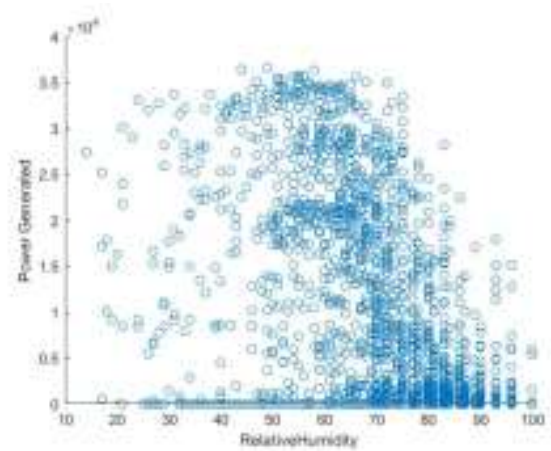

Fig-11. Power Generated v/s Humidity 
International Journal of Current Science Research and Review

ISSN: 2581-8341

Volume 04 Issue 02 February 2021

DOI: 10.47191/ijcsrr/V4-i2-06, Impact Factor: 5.825

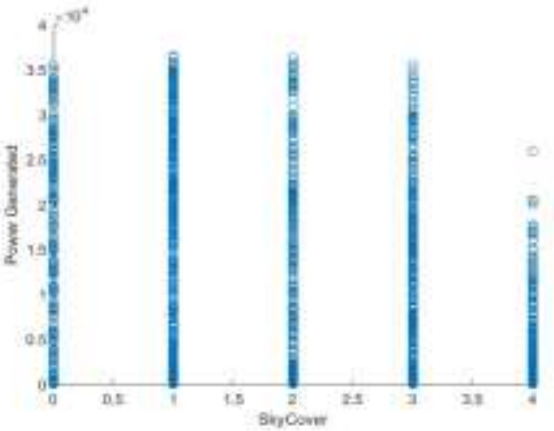

Fig-12. Power Generated v/s Sky Cover

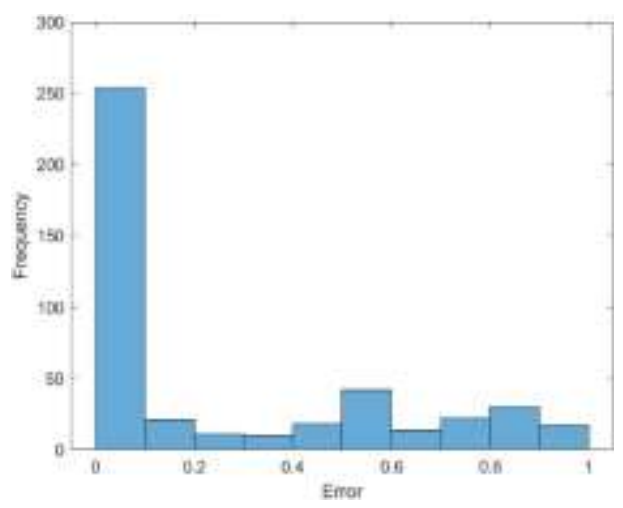

Fig-14. Error v/s Error Frequency After 100 Iteration

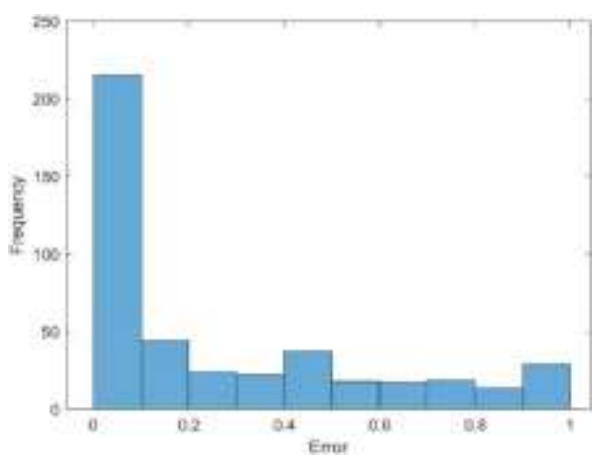

Fig-16. Error v/s Error Frequency After 1000 Iteration

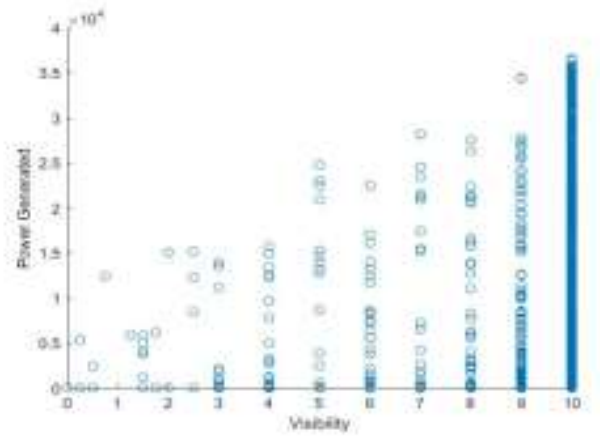

Fig-13. Power Generated v/s Visibility

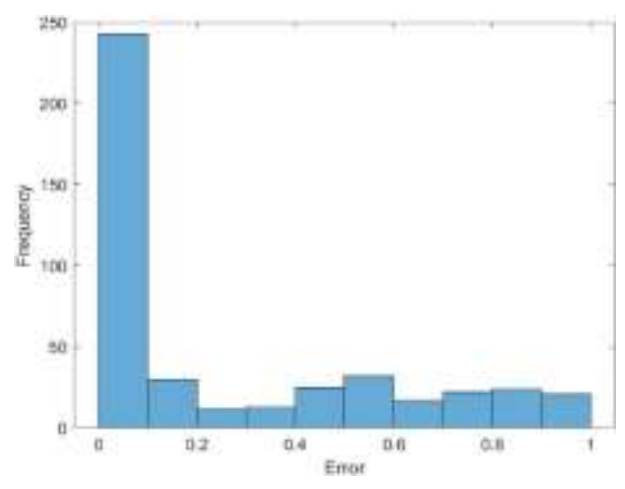

Fig-15. Error v/s Error Frequency After 10 Iteration

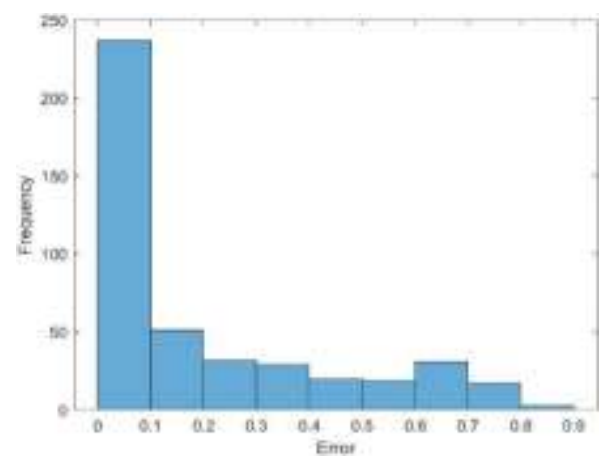

Fig-17. Error v/s Error Frequency After 10000 Iteration

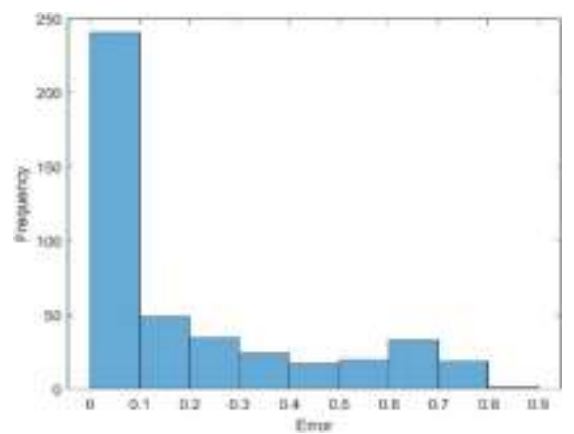

Fig-18. Error v/s Error Frequency after 100000 Iteration 


\section{International Journal of Current Science Research and Review}

ISSN: 2581-8341

Volume 04 Issue 02 February 2021

DOI: 10.47191/ijcsrr/V4-i2-06, Impact Factor: 5.825

IJCSRR @ 2021

www.ijcsrr.org

\section{RESULT AND DISCUSSION \\ Results:}

Results computed in our present work is tabulated below, our findings is highly encouraging.

Table 4.1. Results for Different Iterations

\begin{tabular}{|c|c|c|c|c|c|}
\hline Results computed in our pres & $1^{\text {st }}$ & $2^{\text {nd }}$ & $3^{\text {rd }}$ & $4^{\text {th }}$ & $5^{\text {th }}$ \\
\hline Actual & 0 & 0.218 & 0.771 & 0.907 & 0.571 \\
\hline Prediction 10 iteration & $2.81 \mathrm{e}-22$ & $8.381 \mathrm{e}-21$ & $1.199 \mathrm{e}-14$ & $1.164 \mathrm{e}-06$ & 0.001 \\
\hline Prediction 100 iteration & $3.520 \mathrm{e}-10$ & $6.210 \mathrm{e}-09$ & $9.113 \mathrm{e}-04$ & 0.9998 & 1.0 \\
\hline Prediction1000 iteration & 0.0015 & 0.0092 & 0.9414 & 1.0 & 1.0 \\
\hline Prediction10000 iteration & 0.0282 & 0.0491 & 0.2624 & 0.6810 & 0.7482 \\
\hline Prediction 1000000 iteration & 0.0144 & 0.0235 & 0.2156 & 0.6737 & 0.7671 \\
\hline
\end{tabular}

\section{Discussion:}

Our model can predict the output with low and medium error in the predicted values, but in order to achieve low or negligible error in our model very high number of iterations are required which demands lot of processing time and processing power. We observed that larger the value of learning rate smaller the number of iterations required for training the model although the value of the error in the final trained model is very high. On the contrary smaller the value of learning rate larger the number of iterations required but the error in the final trained model is smaller. In our observation we conclude that higher number of iterations needs to decrease in the number of high value error although the number of low value or medium value are considerably large. Thus, to have a faster and more efficient prediction we must use shallow or deep neural network.

Since very little data is available for the environmental impact on photovoltaic generation therefore it is difficult to draw any special remarks. However, Comparison of presently value with scarcely available experimental and theoretical data is quite encouraging. Further, results computed in our present work will enrich the data bank and our observation to the present investigation may provide guideline to future research in this field.

\section{Future Scope:}

Future scope of the project is to develop shallow and deep neural network which will have higher efficiency and faster learning rate.

\section{REFERENCES}

1. Deng, R.; Chang, N.L.; Ouyang, Z.; Chong, C.M. A, (2019) 'Techno-economic review of silicon photovoltaic module recycling'. Renew. Sustain. Energy Rev. 109, 532-550.

2. D. Agarwal, V. Gupta, D. Jaiswal and A. K. Mandpura, (2020) 'A Machine Learning-Based Approach for PV Power Forecasting', International Conference for Emerging Technology (INCET), Belgaum, India, 1-4.

3. G. Corani, (2005) 'Air quality prediction in Milan: feed-forward neural networks, pruned neural networks and lazy learning', Ecological Modelling, vol. 185, no. 2-4, pp. 513-529.

4. Laurence Stamford and Adisa Azapagic (2018) Energy Technol. 6, 1148-1160.

5. S. Mekhilef, R. Saidur, M. Kamalisarvestani (2012) Renewable and Sustainable Energy Reviews 16(5) $2920-2925$.

6. Ramadan J. Mustafa, Mohamed R. Gomaa, Mujahed Al-Dhaifallah, and Hegazy Rezk (2020) 'Environmental Impacts on the Performance of Solar Photovoltaic Systems', Sustainability, 12(2), 608.

7. Elsner, J. B. and Tsonis, A.A. (1992) 'nonlinear prediction, chaos and noise', Bull Amer. Meteor. Soc., 73, 49-60.

8. C. Zhang, S. Bengio, M. Hardt, B. Recht, and O. Vinyals (2016) 'Understanding deep learning requires rethinking generalization'. International Conference on Learning Representations, November 2016.

9. McCulloch, Warren S.; Pitts, Walter (1943) 'A logical calculus of the ideas immanent in nervous activity', Bulletin of Mathematical Biophysics. 5 (4): 115-133

10. B. Widrow and M. E. Hoff (1960) 'Adaptive Switching Circuits', IRE WESCON Convention Record, pp. 96-104. 


\section{International Journal of Current Science Research and Review}

ISSN: 2581-8341

Volume 04 Issue 02 February 2021

DOI: 10.47191/ijcsrr/V4-i2-06, Impact Factor: 5.825

IJCSRR @ 2021

wWw.ijcsrr.org

11. M. A. AbdulAzeez (2011) 'Artificial neural network estimation of global solar radiation using meteorological parameters in Gusau, Nigeria’, Archives of Applied Science Research, vol. 3, pp. 586-595.

12. C. Chen, S. Duan, T. Cai, and B. Liu (2011) 'Online 24-h solar power forecasting based on weather type classification using artificial neural network', Solar Energy, vol. 85, no. 11, pp. 2856-2870.

13. C. Paoli, C. Voyant, M. Muselli, and M.-L. Nivet (2010) 'Forecasting of preprocessed daily solar radiation time series using neural networks', Solar Energy, vol. 84, no. 12, pp. 2146-2160.

14. A. Linares-Rodr'1guez, J. A. Ruiz-Arias, D. Pozo-Vazquez, and ' J. Tovar-Pescador (2011) 'Generation of synthetic daily global solar radiation data based on ERA-Interim reanalysis and artificial neural networks’, Energy, vol. 36, no. 8, 53565365 .

15. A. Mellit and S. Shaari (2009) 'Recurrent neural network-based forecasting of the daily electricity generation of a Photovoltaic power system', Ecological Vehicle and Renewable Energy (EVER), 26-29, Monte-Carlo, Monaco, March 2009.

16. Corani, G. (2005) 'Air quality prediction in Milan: feed-forward neural networks, pruned neural networks and lazy learning', Ecological Modelling, 185, 513-529.

17. P. Werbos, (1974) 'Beyond regression: New tools for prediction and analysis in the behavioural science', Ph.D. dissertation, committee on Appl. Math., Harward University, Cambridge, MA, Nov. 1974.

18. Parker, David B. (1985) 'Learning-Logic', Technical Report TR-47, Center for Computational Research in Economics and Management Science, MIT, April 1985.

19. Rumelhart, D., Hinton, G. \& Williams, R. (1986) 'Learning representations by back-propagating errors'. Nature 323, 533536.

20. A. V. Timbus, R. Teodorescu, F. Blaabjerg, and U. Borup (2004) 'Online grid measurement and ENS detection for PV inverter running on highly inductive grid', IEEE Power Electronics Letters, vol. 2, no. 3, 77-82.

21. A. Mellit and A. M. Pavan (2010) 'A 24-h forecast of solar irradiance using artificial neural network: application for performance prediction of a grid-connected PV plant at Trieste, Italy', Solar Energy, vol. 84, no. 5, 807-821.

22. Kamarthi, S.V. and Pittner, S, (1999) 'Accelerating neural network training using weight extrapolation', Neural Network, $12,1285-1299$.

23. F. O. Hocaoglu, ` O. N. Gerek, and M. Kurban (2008) 'Hourly solar radiation forecasting using optimal coefficient 2-D linear filters and feed-forward neural networks', Solar Energy, vol. 82, no. 8, 714-726.

24. K. A. Kumari, N. K. Boiroju, T. Ganesh, and P. R. Reddy (2012) 'Forecasting surface air temperature using neural networks', International Journal of Mathematics and Computer Applications Research, vol. 3, 65-78.

25. T. Khatib, A. Mohamed, K. Sopian, and M. Mahmoud, (2012) 'Solar energy prediction for Malaysia using artificial neural networks', International Journal of Photoenergy, vol. 2012, Article ID 419504.

26. Nielsen, M. (2015) 'Neural Networks and Deep Learning', Determination Press.

27. Aurélien Géron (2019) 'Hands-on Machine Learning with Scikit-Learn, Keras, and Tensor Flow', O’ Reilly Media, 600 pp.

Cite this Article: Shreeniket Trivedi (2021). Evaluation of the Use of Artificial Neural Networks to Predict the Photovoltaic Power Generation Factors by Using Feed Forward Back Propagation (FFBP) Technique . International Journal of Current Science Research and Review, 4(2), 113-119 